\title{
Magda Heras 2021 Merecido destaque engalana nuestra revista en tiempos de pandemia
}

\author{
Dr. Federico Ferrando
}

\section{El premio Magda Heras}

Magda Heras nació en Terrassa en 1953. Tras estudiar Medicina en la Universidad de Barcelona, se formó primero en Medicina Interna, en el Hospital Mútua de Terrassa, y luego en Cardiología, en el Hospital Clínic de Barcelona. En 1986, se doctoró en la Universidad de Barcelona. Luego, fue investigadora del Laboratorio de Trombosis y Arteriosclerosis de la Clínica Mayo en Rochester (Minnesota, EE.UU.). A su regreso a España, se incorporó al Servicio de Cardiología del Hospital Clínic de Barcelona, donde desarrolló una prolífica trayectoria asistencial y científico-académica: publicó más de 200 artículos y 27 capítulos de libro y dirigió 7 tesis doctorales. En 2005 fue nombrada Profesora Asociada de Cardiología de la Universidad de Barcelona. Tuvo una colaboración muy activa con la Sociedad Europea de Cardiología e integró diferentes grupos de trabajo. En octubre de 2009, fue nombrada editora jefe de la Revista Española de Cardiología (REC). Tras dedicar gran parte de su tiempo a esta publicación, en particular durante sus últimos años, la Dra. Heras fallece el 3 de agosto de $2014^{(1)}$.

Detrás del legado de Magda hay una historia de hermandad entre la Sociedad Española de Cardiología (SEC) y la Sociedad Uruguaya de Cardiología (SUC). En 2013, a propuesta de la $R E C$, con Magda Heras a la cabeza, junto al comité editorial de los Archivos de Cardiología de México, el entonces editor jefe de nuestra Revista Uruguaya de Cardiología (RUC), Dr. Walter Reyes Caorsi, actuó como coordinador de la Red Iberoamericana de Revistas Cardiovasculares. Esta iniciativa estuvo dirigida principalmente a fomentar los vínculos interinstitucionales y potenciar la difusión y visibilidad de las publicaciones de la $R U C$. Entre sus logros estuvo el lanzamiento del Premio Magda Heras, una distinción anual al mejor trabajo original de las revistas cardiológicas iberoameri- canas, que implica, desde su generación, un reconocimiento de calidad científica internacional y un premio en metálico para los investigadores, financiado por la $R E C$ y la Sociedad Interamericana de Cardiología ${ }^{(2)}$. A su vez, el galardón reconoce el gran trabajo de Magda en la REC desde 2005 hasta su partida en 2014. La selección de los mejores trabajos dignos de premio se realiza mediante una convocatoria abierta, en la que cada equipo editorial presenta como candidato el artículo que considera más relevante publicado en las ediciones del período anual correspondiente.

En su IX edición 2021, el Premio Magda Heras reconoció la calidad de unas pocas investigaciones desarrolladas y difundidas en 2020, cuando la expansión global de la enfermedad por el SARSCoV2 (COVID19) transformaba por completo la forma en la que se practica la Medicina y el avance de la Ciencia en materia de salud humana.

\section{Los artículos premiados en la IX edición del} Magda Heras (2021). Un pequeño infiltrado entre los grandes

Las revistas asociadas al premio sometieron los artículos preseleccionados a votación en el consejo editorial, mediante una lista de cotejo diseñada ad hoc, con escalas de juicios del 1 al 10 en los subítems: Importancia del tema, Metodología, Adecuación de los resultados (estadística), Calidad de la discusión y Resultados. Los puntajes promedio de estos juicios fueron agregados desde la $R E C$ (tabla 1), obteniéndose una puntuación final para cada artículo que justificaba el fallo.

Desde su primera entrega en 2013, la posición de podio en el Magda Heras había sido elusiva para los artículos publicados en la $R U C$. En las tres últimas ediciones (2017-2019), la $R E C$ estuvo en lo más alto del podio en dos instancias (2017 y 2018) y en tercer lugar en 2019, mientras que 


\begin{tabular}{|c|c|c|c|c|c|c|}
\hline $\mathrm{n} .^{\circ}$ & $\begin{array}{l}\text { Sociedad } \\
\text { Científica }\end{array}$ & $\begin{array}{l}\text { Autor } \\
\text { principal }\end{array}$ & Institución & Título & Diseño & Publicado en \\
\hline 1 & $\begin{array}{l}\text { Sociedad Chilena } \\
\text { de Cardiología } \\
\text { (Sochicar) }\end{array}$ & A. Loniz & $\begin{array}{l}\text { Pontificia Universidad } \\
\text { Católica de Chile }\end{array}$ & $\begin{array}{l}\text { Remodelado cardíaco en el modelo } \\
\text { preclínico de falla cardíaca isquémica } \\
\text { experimental por dislipidemia en el } \\
\text { ratón SR-B1 KO/ApoER } 6 \text { 1h/h }\end{array}$ & Experimental & $\begin{array}{l}\text { Rev Chil Cardiol. } \\
\text { 2020; } 39 \text { (1): } 2433\end{array}$ \\
\hline 2 & $\begin{array}{l}\text { Asociación Nacional } \\
\text { de Cardiólogos de } \\
\text { México (ANCAM) }\end{array}$ & $\begin{array}{l}\text { R. Robledo } \\
\text { Nolasco }\end{array}$ & $\begin{array}{l}\text { Centro Médico Nacional } \\
20 \text { de Noviembre del } \\
\text { ISSSTE }\end{array}$ & $\begin{array}{l}\text { Radiofrequency catheter ablation of } \\
\text { cardiac arrhythmias using only three } \\
\text { dimensional mapping systems }\end{array}$ & Serie de casos & $\begin{array}{l}\text { Cardiovasc Metab Sci. } \\
\text { 2020; } 31 \text { (1): } 48\end{array}$ \\
\hline 3 & $\begin{array}{l}\text { Sociedad Cubana de } \\
\text { Cardiología }\end{array}$ & $\begin{array}{l}\text { R. Carmona } \\
\text { Puerta }\end{array}$ & $\begin{array}{l}\text { Hospital Universitario } \\
\text { Cardiocentro Ernesto } \\
\text { Guevara }\end{array}$ & $\begin{array}{l}\text { Duración de la onda P en las } 12 \\
\text { derivaciones del electrocardiograma } \\
\text { y su relación con el tiempo de } \\
\text { conducción interauricular en } \\
\text { pacientes con taquicardias } \\
\text { paroxísticas supraventriculares }\end{array}$ & $\begin{array}{l}\text { Observacional } \\
\text { transversal }\end{array}$ & $\begin{array}{l}\text { CorSalud. } \\
\text { 2020; } 12 \text { (3): } 24753\end{array}$ \\
\hline 5 & $\begin{array}{l}\text { Sociedad Española de } \\
\text { Cardiología }\end{array}$ & $\begin{array}{l}\text { O. Rodríguez- } \\
\text { Leor }\end{array}$ & $\begin{array}{l}\text { Grupo de Trabajo } \\
\text { sobre Código Infarto, } \\
\text { Asociación } \\
\text { de Cardiología } \\
\text { Intervencionista de la } \\
\text { Sociedad Española de } \\
\text { Cardiología }\end{array}$ & $\begin{array}{l}\text { Impacto de la COVID19 en el } \\
\text { tratamiento del infarto agudo de } \\
\text { miocardio con elevación del segmento } \\
\text { ST. La experiencia española }\end{array}$ & $\begin{array}{l}\text { Cohortes } \\
\text { retrospectivo } \\
\text { multicéntrico }\end{array}$ & $\begin{array}{l}\text { Rev Esp Cardiol. } \\
\text { 2020; } 73 \text { (12): } 9941002\end{array}$ \\
\hline 6 & $\begin{array}{l}\text { Sociedad Española de } \\
\text { Cardiología }\end{array}$ & $\begin{array}{l}\text { O. Rodríguez- } \\
\text { Leor }\end{array}$ & $\begin{array}{l}\text { Participantes del } \\
\text { Registro de Código } \\
\text { Infarto de la ACI-SEC }\end{array}$ & $\begin{array}{l}\text { Impacto de la pandemia de COVID19 } \\
\text { sobre la actividad asistencial en } \\
\text { cardiología intervencionista en } \\
\text { España }\end{array}$ & $\begin{array}{l}\text { Encuesta } \\
\text { telemática nacional }\end{array}$ & $\begin{array}{l}\text { REC Interv Cardiol. } \\
\text { 2020; } 2 \text { (2): } 829\end{array}$ \\
\hline 8 & $\begin{array}{l}\text { Sociedad Argentina } \\
\text { de Cardiología }\end{array}$ & M. I. Figueroa & $\begin{array}{l}\text { Instituto de Ecorregiones } \\
\text { Andinas (INECOA) } \\
\text { UNJu-CONICET. } \\
\text { Departamento de } \\
\text { Biología Molecular- } \\
\text { Instituto de Estudios } \\
\text { Celulares, Genéticos y } \\
\text { Moleculares (ICeGeM) } \\
\text { UNJu }\end{array}$ & $\begin{array}{l}\text { Distribución del riesgo cardiovascular } \\
\text { en la Argentina en } 2018\end{array}$ & $\begin{array}{l}\text { Prevalencia } \\
\text { comparativa de } \\
\text { perfiles de riesgo }\end{array}$ & $\begin{array}{l}\text { Rev Argent Cardiol. } \\
\text { 2020; } 88 \text { (4): } 31723\end{array}$ \\
\hline 9 & $\begin{array}{l}\text { Instituto Nacional } \\
\text { de Cardiología } \\
\text { Ignacio Chávez, } \\
\text { Sociedad Mexicana } \\
\text { de Cardiología }\end{array}$ & $\begin{array}{l}\text { A. Abundes- } \\
\text { Velasco }\end{array}$ & $\begin{array}{l}\text { Instituto Nacional de } \\
\text { Cardiología Ignacio } \\
\text { Chávez }\end{array}$ & $\begin{array}{l}\text { Diseño y fabricación del stent } \\
\text { coronario INC-1 y pruebas iniciales } \\
\text { en animales de experimentación }\end{array}$ & $\begin{array}{l}\text { Opinión de } \\
\text { expertos- } \\
\text { descripción de } \\
\text { diseño y desarrollo } \\
\text { tecnológico }\end{array}$ & $\begin{array}{l}\text { Arch Cardiol Méx. } \\
\text { 2020; } 90 \text { (2): } 1638\end{array}$ \\
\hline 10 & $\begin{array}{l}\text { Sociedad Uruguaya } \\
\text { de Cardiología }\end{array}$ & D. Pérez Zerpa & $\begin{array}{l}\text { Instituto Nacional de } \\
\text { Cirugía Cardíaca }\end{array}$ & $\begin{array}{l}\text { Perfil clínico y etiológico de pacientes } \\
\text { operados con endocarditis activa. } \\
\text { Seguimiento a diez años }\end{array}$ & $\begin{array}{l}\text { Cohortes } \\
\text { retrospectivo } \\
\text { unicéntrico }\end{array}$ & $\begin{array}{l}\text { Rev Urug Cardiol. } \\
\text { 2020; } 35 \text { (2): } 16979\end{array}$ \\
\hline 11 & $\begin{array}{l}\text { Sociedad Colombiana } \\
\text { de Cardiología }\end{array}$ & D. I. Molina & $\begin{array}{l}\text { Universidad de Caldas, } \\
\text { Asociación IPS médicos } \\
\text { internistas de Caldas }\end{array}$ & $\begin{array}{l}\text { Inhibidores de la enzima convertidora } \\
\text { de angiotensina y bloqueadores de } \\
\text { los receptores de angiotensina II: } \\
\text { ¿aumentan el riesgo de padecer } \\
\text { COVID19? }\end{array}$ & $\begin{array}{l}\text { Revisión no } \\
\text { sistemática }\end{array}$ & $\begin{array}{l}\text { Rev Colomb Cardiol. } \\
\text { 2020; } 27 \text { (3): } 1326\end{array}$ \\
\hline 12 & $\begin{array}{l}\text { Federación Argentina } \\
\text { de Cardiología }\end{array}$ & E. Frontera & $\begin{array}{l}\text { Integrantes del } \\
\text { Comité de Cardiopatía } \\
\text { Isquémica de FAC y de } \\
\text { los investigadores del } \\
\text { Registro ARGEN-IAM }\end{array}$ & $\begin{array}{l}\text { Enfermedad de múltiples vasos en } \\
\text { pacientes con infarto de miocardio con } \\
\text { elevación del segmento ST. Evolución } \\
\text { y complicaciones intrahospitalarias. } \\
\text { (Registro ARGEN-IAM). }\end{array}$ & $\begin{array}{l}\text { Registro } \\
\text { multicéntrico }\end{array}$ & $\begin{array}{l}\text { Rev Fed Arg Cardiol. } \\
\text { 2020; } 49 \text { (1): } 148\end{array}$ \\
\hline
\end{tabular}

Archivos de Cardiología de México logró la tercera mención en 2017 y el segundo puesto en 2019. En un logro sin precedentes, un artículo publicado en la $R U C$ durante 2020 recibió la mención al tercer premio 2021, solo superado por dos trabajos de renombradas revistas de habla hispana, voceras de sociedades con gran capacidad de difusión e impacto científico, como la REC y la Revista Chilena de Cardiología. Para una sociedad científica pequeña, con poco más de seiscientos socios, mí- 


\begin{tabular}{|c|c|c|c|c|c|c|c|}
\hline Premio & $\begin{array}{l}\text { Sociedad } \\
\text { científica- } \\
\text { revista }\end{array}$ & $\begin{array}{l}\text { Edad } \\
\text { (años) }\end{array}$ & $\begin{array}{l}\text { Núm. } \\
\text { socios }\end{array}$ & $\begin{array}{l}\text { Revista-núm. } \\
\text { artículos anuales } \\
\text { en } 2020\end{array}$ & $\begin{array}{l}\text { Revista-núm. } \\
\text { investigaciones } \\
\text { originales en } 2020 \text {, } \\
\text { n (\%) }\end{array}$ & $\begin{array}{l}\text { Revista-Bases y repositorios } \\
\text { (indexación) }\end{array}$ & $\begin{array}{l}\text { Revista- } \\
\text { FI (2020) }\end{array}$ \\
\hline $1 .^{\circ}$ & SEC-REC & 77 & +4000 & 382 & $59(15 \%)$ & $\begin{array}{l}\text { Journal Citation Reports and } \\
\text { Science Citation Index Expanded, } \\
\text { Current Contents, MEDLINE, Index } \\
\text { Medicus, Embase, Excerpta Medica } \\
\text { ScienceDirect, Scopus }\end{array}$ & 4.753 \\
\hline $2 .^{\circ}$ & $\begin{array}{l}\text { Sochicar- } \\
\text { Rev. Chilena de } \\
\text { Cardiología }\end{array}$ & 73 & $+700 \ddagger$ & 33 & $6(18 \%)$ & Scielo, Latindex, Redalyc & $\begin{array}{l}\text { No } \\
\text { disponible }\end{array}$ \\
\hline $3 .^{\circ}$ & SUC-RUC & 73 & +600 & 59 & $12(20 \%)$ & Scielo, Latindex, Redalyc & $\begin{array}{l}\text { No } \\
\text { calculado }\end{array}$ \\
\hline $\begin{array}{l}\ddagger \text { Datos } \\
\text { FI: facto } \\
\text { guaya } d\end{array}$ & $\begin{array}{l}\text { sponibles al añ } \\
\text { de impacto. SE } \\
\text { Cardiología. Rl }\end{array}$ & $\begin{array}{l}2018 . \\
\text { C: Socie } \\
\text { C: Rev }\end{array}$ & $\begin{array}{l}\text { Espa } \\
\text { Españ }\end{array}$ & $\begin{array}{l}\text { de Cardiología. } \\
\text { e Cardiología. } \mathrm{R}\end{array}$ & $\begin{array}{l}\text { chicar: Sociedad } \\
\text { C: Revista Urugu }\end{array}$ & $\begin{array}{l}\text { tilena de Cardiología. SUC: Socied } \\
\text { ya de Cardiología. }\end{array}$ & lad Uru- \\
\hline
\end{tabular}

nimos recursos dedicados a la gestión y una gran carga de trabajo honorario, esto significa algo así como colarse entre dos grandes (tabla 2). La SEC, fundada en Madrid en 1944, cuenta con más de 4.000 socios, entre cardiólogos y profesionales relacionados con las enfermedades cardiovasculares, tanto españoles como procedentes del ámbito europeo, latinoamericano e internacional. Esta Sociedad gestiona tres revistas en su familia de publicaciones (tabla 1). La más difundida y prestigiosa de ellas es la $R E C$, que integra la macrorred de publicaciones científicas de la Sociedad Europea de Cardiología (ESC National Societies Cardiovascular Journals Editors' Network), y está indexada en bases de datos y repositorios de gran difusión como MEDLINE, Embase y Scopus, entre otros. Para 2020, la REC publicaba un número mensual que incluía un promedio de 5 artículos originales cada uno. En este mismo año, superó un factor de impacto de 4,7 según Clarivate Analytics-Journal Citation Reports 2021; llega a esta posición escalonando en forma sostenida y casi continua desde 2011 (2,53), y transformándose en una de las 100 revistas europeas mejor posicionadas en la disciplina de acuerdo con el ranking Scimago 2020 (tercer cuartil). La Revista Chilena de Cardiología es la publicación oficial de la Sociedad de Cardiología y Cirugía Cardiovascular de Chile (Sochicar), una institución que coordina, ejecuta y difunde actividades científicas y de promoción de salud desde hace 73 años. La Sochicar, que ya contaba más de setecientos socios para 2018, trabaja con diversas disciplinas afines en tres filiales. Aunque las tres Sociedades Científicas premiadas en esta IX edición 2021 a través de sus revistas tienen una edad similar, la Revista Chilena de Cardiología podría ser más comparable a nuestra $R U C$, al menos en algunos aspectos de escala y contenidos (tabla 2).

El primer premio del Magda Heras 2020 fue otorgado a una gran investigación epidemiológica española publicada en la $R E C$. Sus hallazgos principales representan un aporte mayor al estudio de los impactos de la pandemia COVID-19 en Europa. La experiencia, titulada: "Impacto de la COVID-19 en el tratamiento del infarto agudo de miocardio con elevación del segmento ST. La experiencia española”, fue publicada por O. Rodríguez-Leor y col. ${ }^{(3)}$. En dicha experiencia los autores analizan los cambios en el tratamiento del IAMCEST durante el primer brote de COVID-19. Con base en un registro multicéntrico nacional de pacientes, que fueron atendidos consecutivamente, comparan características de los pacientes, procedimientos y resultados hospitalarios obtenidos en 2 cohortes, tratadas antes o durante la COVID-19. Los autores encontraron que las redes de IAMCEST de 75 hospitales atendieron a un total de 1.113 pacientes durante el brote de COVID-19 frente a los 1.538 tratados en el mismo período del año anterior, lo cual representa una reducción del $27,6 \%$, mientras que los pacientes con IAMCEST confirmado se redujeron en $22,7 \%$. No hubo diferencias en la estrategia de reperfusión adoptada (muy mayoritariamente mediante intervencionismo percutáneo primario). El tiempo de isquemia fue más prolongado durante el período de pandemia, sin diferencias en el componente temporal primer contacto médico-reperfusión ("menos pacientes con IAMCEST y mayores demoras hasta la reperfusión”). La mortalidad hospitalaria, considerada como objetivo 
primario, fue mayor durante la COVID-19 (7,5 frente al 5,1\%), aunque esta asociación no se mantuvo tras ajustar por otros factores de confusión (tiempo desde el inicio de los síntomas a la reperfusión, edad, sexo, clase de Killip y resultado positivo de rtPCR para COVID-19). Los investigadores evaluaron la robustez de estos resultados mediante dos análisis de sensibilidad, demostrando que la COVID-19 fue, muy probablemente, el factor que determinó el aumento de mortalidad hospitalaria evidenciado tras comparar ambas cohortes. Entre los pacientes tratados durante el brote de COVID-19, la incidencia de infección confirmada fue baja (6,3\%). Los autores concluyen que, durante el brote de COVID-19, existió una disminución en el número de pacientes con IAMCEST, una prolongación del tiempo dolor-reperfusión y un aumento en la mortalidad hospitalaria. Se trata de un gran estudio observacional de cohortes retrospectivo, multicéntrico, de diseño riguroso e impecable análisis multivariado que, evaluando las intervenciones documentadas en el Registro Código Infarto, permitió cuantificar los impactos del primer brote de COVID-19 sobre el tratamiento del IAMCEST en toda España. Dicha experiencia proveyó información epidemiológica de primera mano que fue destacada por las sociedades cardiológicas europeas ${ }^{(5)}$.

El segundo premio fue destinado a una investigación básica titulada: "Remodelado cardíaco en el modelo preclínico de falla cardíaca isquémica experimental por dislipidemia en el ratón SR-B1 KO/ApoER6 1h/h", publicada por A. Lonis y col. de la Universidad Católica de Chile en la Revista Chilena de Cardiología(4). En esta investigación básica, los autores caracterizan el remodelado miocárdico en un modelo animal de falla cardíaca severa de etiología isquémica, comparando los cambios estructurales e histopatológicos, la evolución de la función contráctil y la sobrevida asociada a la enfermedad cardíaca por ateromatosis dietéticamente inducida con un grupo control. Este modelo animal se induce sometiendo a ratones homocigotos SR-B1 KO/ApoER6 1h/h a una dieta aterogénica durante 8 semanas. En los especímenes postmortem, el remodelado miocárdico de los ratones homocigotos se caracterizó por intensa apoptosis y fibrosis, con un aumento de la fibrosis cardíaca de 7,9 veces y del número de cardiomiocitos apoptóticos de casi 56 veces. Dichos cambios, que no habían sido cuantificados previamente en este modelo experimental, sugieren fuertemente que el proceso de apoptosis juega un rol importante en el deterioro de la función sistólica, la falla cardíaca y la mortalidad. A su vez, dichos procesos se asociaron con un aumento discreto de los infiltrados de macrófagos en la pared cardíaca. En lo estructural y funcional, se observó dilatación cavitaria y disminución de la FEVI a las 4 semanas, en contraste con el grupo control. Los investigadores también detectaron un aumento de la masa cardíaca relativa (de 2,1 veces) y del peso pulmonar relativo (en $80 \%$ ), sin cambios en las dimensiones de los cardiomiocitos. Dichos cambios anatómico-estructurales sugieren que el edema parietal también podría jugar un papel relevante en este proceso. La supervivencia en el grupo de ratones con dieta aterogénica se redujo en $46,7 \%$ y estuvo dada, en primer lugar, por disminución progresiva de la función sistólica y deterioro severo del bienestar animal. La otra causa principal de muerte en este modelo fue la muerte súbita, aunque no se pudo establecer con precisión si esto se debió al desarrollo de arritmias malignas o a la falla cardíaca rápidamente progresiva, para lo cual se requieren métodos de observación y monitoreo más precisos. Esta experiencia chilena, de excelente concepción y diseño, arroja nueva información experimental sobre la fisiopatología e impactos anátomo-estrucurales de la enfermedad ateroesclerótica, incluso sugiriendo potenciales blancos patogénicos para el desarrollo de nuevas terapias.

En el artículo que obtuvo el tercer premio, D. Pérez Zerpa y col. del Instituto Nacional de Cirugía Cardíaca analizan con rigurosidad el pronóstico de los pacientes con endocarditis infecciosa (EI) que requieren procedimientos quirúrgicos, desde la perspectiva de un equipo de cardiólogos y cirujanos cardíacos ${ }^{(5)}$. El autor analiza de manera retrospectiva los resultados quirúrgicos obtenidos en una población de 101 pacientes intervenidos, evaluando datos evolutivos a largo plazo (10 años). Reportan una mortalidad global operatoria de $19,8 \%$, que es mayor (casi $30 \%$ ), en presencia de complicaciones locales, principalmente abscedación. La sobrevida global a 5 y 10 años fue de $88,2 \%$ y $81,5 \%$, respectivamente. Tras un análisis de regresión logistica (Cox), se demuestra que la mortalidad a largo plazo fue significativamente superior en aquellos pacientes que presentaban complicaciones locales y vegetaciones ubicadas en el velo posterior de la válvula mitral. Otras variables, como la duración del tratamiento antibiótico prequirúrgico o la presencia de fiebre al momento de esta, no fueron predictores independientes para eventos adversos. Estos resultados son similares a lo reportado en los grandes estudios observacionales disponibles. Desde el punto de vista bacteriológico y epidemiológico, se destaca una alta incidencia de agentes intrahospitalarios 
y procedimientos invasivos como probables predisponentes patogénicos. A su vez, la presencia de vegetaciones sobre el velo posterior mitral y la infección por Staphylococcus aureus fueron predictores independientes para el desarrollo de embolias sistémicas. Con base en estos hallazgos locales, los autores sugieren que aquellos pacientes que cursan EI con complicaciones locales deben ser seguidos de cerca, incluso por períodos prolongados, ya que esta variable representa el principal predictor de muerte durante el seguimiento. Además, la tasa de procedimientos previos asociados al desarrollo de EI y la caracterización de la flora responsable permite planificar diversas acciones preventivas hospitalarias que podrían impactar de manera positiva sobre la morbimortalidad. Aunque unicéntricos, creemos que estos hallazgos locales proveen información de gran valor clínico al cardiólogo, equipo quirúrgico actuante y todos los profesionales en salud que abordan los pacientes con EI. La disponibilidad de información evolutiva relevante, con seguimiento durante una década, y el sólido análisis multivariado son otras fortalezas para subrayar y aplaudir de la experiencia de D. Pérez Zerpa y col. Bien sabido es que se requieren bases de datos extensas y adecuadamente actualizadas para aplicar un análisis crítico y riguroso sobre datos evolutivos, lo que resulta una excepción más que la regla en el medio. En tiempos de pandemia, este consejo editorial felicita a todos los autores de dicha experiencia, que enaltecen a la SUC a través de la $R U C$.

\section{Un contexto único para las sociedades y revistas científicas}

La investigación científica no se había visto interrumpida de una forma tan amplia y radical desde la Segunda Guerra Mundial. La práctica de la Medicina, a todos los niveles y en todos los ámbitos, afrontó enormes desafios, y el trabajo de los científicos se transformó de forma radical. En general, la comunidad científica respondió a la crisis sanitaria con una amplia movilización de todos sus recursos, en un intento por arrojar luz sobre las características del nuevo coronavirus, sus mecanismos de transmisión, patogenia, aspectos clínicos estrategias de prevención y manejo terapéutico. Casi tan pronto como se descubrió el agente SARS-Co-V2, los grupos de investigación básica exploraron a fondo su biología, mientras que otros desarrollaron pruebas de uso diagnóstico (amplificación de ADN viral mediante rtPCR, detección de antígenos, caracterización de la respuesta inmune mediante determinación de an- ticuerpos específicos), y ensayaron y midieron el impacto de diversas medidas de Salud Pública, en un intento por controlar los contagios. Los científicos también diseñaron y ejecutaron experiencias dirigidas a desarrollar nuevas terapias y vacunas que fueran seguras y efectivas. La ciencia jamás había progresado tan rápido en respuesta a ningún otro agente infeccioso.

En opinión de la mayoría, las revistas científicas respondieron de forma casi inmediata, abierta y eficaz. Los sistemas de difusión de la información médica se vieron afectados por un aluvión de artículos publicados, procesos de revisión acelerados, medios diseñados para un acceso fácil y efectivo, mayor atención a las plataformas con formatos de preimpresión (preprints) y posibilidad de acceso gratuito a casi todos los artículos relacionados con la pandemia ${ }^{(6)}$. El número de artículos que surgió de ensayos clínicos y estudios observacionales se multiplicó a un ritmo sin precedentes. Durante abril de 2020, varios gigantes de la publicacion cientifica médica como JAMA, The Lancet, The British Medical Journal, New England Journal of Medicine, todos con factores de impacto siderales, llegaron a tener tirajes casi exclusivamente destinados a la temática COVID-19, desplazando la atención de otras áreas como la oncología clínica, las ciencias básicas, la imagenología o las terapias específicas, por ejemplo. Revistas como Nature y Science llegaron a dedicar una alta proporción de sus artículos a la temática. La red JAMA Network instauró un espacio de puesta al día exclusivamente dedicado a la enfermedad dirigido a sus lectores (Coronavirus Resource Center), con diversas secciones de frecuencia semanal como investigaciones originales, artículos de opinión, revisiones, noticias y reflexiones, humanidad, forum en salud, áreas de disciplinas (como neurología y oncología, por ejemplo), una selección de los artículos mas leídos e información para los pacientes con COVID-19. Desde enero de 2020 a la fecha, solo esta sección de JAMA ya había difundido más de 870 investigaciones originales, 720 artículos de opinión y casi 50 revisiones dedicadas a COVID-19. Este tipo de iniciativas fueron casi la regla entre las revistas médicas de mayor impacto durante 2020.

El número de publicaciones referidas a COVID1-9 y su impacto científico durante los primeros 3 meses de la pandemia fue revisado por C. E. Diéguez-Campa y col. El autor identificó 2.530 publicaciones sobre COVID-19 registradas en la gran base de datos PubMed/MEDLINE, escritas por autores de 67 países (el 57,7\% de China y EE.UU.), que posteriormente generaron más de 59.000 citas. Para 
entonces, a nivel mundial existía una clara correlación entre el número de publicaciones por país y el número de casos confirmados y muertes relacionadas con la enfermedad ${ }^{(7)}$. También se constató un tiempo de revisión más breve y un período más corto desde el envío de los manuscritos hasta el fallo de aceptación o rechazo. En Europa, la mayoría de las revistas de cardiología publicaron varias revisiones referidas a las manifestaciones cardiovasculares de COVID-19, aunque también se difundieron investigaciones originales centradas en campos inusuales que fueron surgiendo durante los primeros brotes ${ }^{(8)}$. Este aumento en la presentación de investigaciones clínicas en cardiología podría tener relación, entre otros factores, con las restricciones a los viajes, la cancelación de eventos médicos, la reducción de reuniones clínicocientíficas y consejos asesores ${ }^{(9)}$. En nuestra región, en cambio, la cantidad de información publicada sobre el COVID-19 fue mucho más limitada. A través de una revisión de la literatura en PubMed, LILACS y los repositorios de preprints bioRxiv y medRxiv, D. Forero-Peña y col. recuperaron y analizaron los documentos disponibles sobre COVID-19 en Latinoamérica entre enero y abril de 2020, excluyendo comentarios editoriales y opiniones de expertos. Los autores encontraron un total de 29 publicaciones, la mayoría provenientes de Brasil (34,4\%), seguido de Colombia y México (20,6\% cada uno), con un enfoque predominantemente epidemiológico $(72,4 \%)^{(10)}$.

La situación mundial indujo a que casi toda la atención, incluyendo la disponibilidad de fondos, la preocupación de los políticos y administradores públicos y privados y los medios de comunicación se focalizara en temas vinculados a la infección por el SARS-CoV-2, sus múltiples aristas epidemiológicas, la patogenia de la infección viral y el potencial desarrollo de terapias específicas y medidas de prevención de contagios. Dicho de otra forma, la investigación relacionada con $\mathrm{CO}$ VID-19 casi monopolizó el enfoque de las publicaciones científicas y los organismos y empresas que financian las investigaciones, en detrimento de otros temas que los equipos editoriales y las agencias ansiaban y debían cubrir.

Como consecuencia directa, muchas áreas, disciplinas, grupos científicos, empresas y centros asistenciales y/o experimentales vieron desplazados y aplazados sus programas de financiamiento en investigacion clínica, básica, epidemiológica y aplicada, en relación directa con el impacto productivo y económico gigantesco que impuso la pandemia. Miles de investigadores que trabajaban en áreas del conocimiento sin relación con el nuevo coronavirus o sus impactos (la gran ma- yoría) vieron retrasados sus proyectos o dejaron sus carreras en suspenso, mientras los fondos disponibles para sus actividades se interrumpían de manera abrupta. A medida que el virus se filtraba a lo largo de las fronteras, un gran número de universidades cerraron sus campus. Los laboratorios detuvieron sus experimentos, excepto aquellos que consideraban más esenciales. Las tareas prácticas (de campo) se suspendieron, y las conferencias, congresos, jornadas, meetings y otros tipos de encuentros científicos pasaron a celebrarse por completo en modalidad virtual. Aunque con gran heterogeneidad según los países y regiones, en general los investigadores se vieron obligados, de repente, a trabajar desde su domicilio, viendo cómo sus vidas cambiaban de forma radical, a menudo luchando para cuidar de su núcleo familiar con acceso limitado a muchos recursos ${ }^{(11)}$. La mayoría de los estudiantes tampoco pudieron acceder a los datos empíricos que precisaban para completar sus proyectos de grado, obtener créditos o títulos. Los cierres de fronteras fueron una barrera adicional para la búsqueda de empleo de muchos científicos. También se acentuaron y desnudaron una serie de disparidades. Algunos grupos de investigadores particularmente afectados fueron las mujeres, los padres y madres con niños pequeños a cargo, los investigadores novatos, los integrantes de universidades u otras instituciones con escasos recursos para la investigación y los científicos que integraban grupos históricamente subrepresentados, para quienes la pandemia magnificó una serie de obstáculos que ya dificultaban una dedicación plena y fulltime a la ciencia. En Brasil, las mujeres afrodescendientes y las madres de todas las etnias reportaron la mayor reducción en su productividad científica, medida por su capacidad para presentar trabajos de investigación y cumplir con los plazos estipulados en proyectos financiados ${ }^{(12)}$. A su vez, las mujeres responsabilizadas de cuidar a sus niños experimentaron una disminución sustancial en el tiempo que podían dedicar a la investigación ${ }^{(13)}$. El aumento en el número de trabajos de investigación presentados tampoco se distribuyó de manera uniforme entre hombres y mujeres, lo que podría ampliar las disparidades de género existentes en los trabajadores de la ciencia, dañando más las perspectivas profesionales futuras de las mujeres.

Aunque un horizonte común de recursos y esfuerzos debidamente unificados para enfrentar el desafío de la pandemia debería haber "iluminado" el camino de la ciencia, hubo un gran número de ensayos clínicos registrados cuya calidad metodológica era (y es) al menos cuestionable. En un 
reciente artículo publicado en The Lancet, J. J. H. Park y col. discuten cómo respondió la Ciencia a la pandemia, enfrentada a una increíble presión sobre diversos actores como los propios investigadores, los organismos reguladores, agencias financiadoras y administradores de políticas. Con ejemplos ilustrativos, y pasando revista a algunos registros de ensayos clínicos, los autores arguyen que la respuesta de la comunidad científica a la pandemia desnudó muchos problemas fundamentales que ya existían en los ensayos clínicos, bajo el sistema actual y sus estructuras de incentivos ${ }^{(14)}$. Comentan que el contexto volvió a enfatizar la importancia de garantizar el desarrollo de ensayos clínicos aleatorizados a gran escala correctamente estructurados, siguiendo protocolos rigurosos, que integren actividades bien coordinadas y colaborativas. Más aún: se subraya la necesidad de optimizar las estructuras y métodos de incentivos para permitir un intercambio más efectivo de datos anónimos, y para brindar oportunidades similares en países y regiones cuyas condiciones sanitarias, sociales y económicas para la investigación recibe una atención menor. Como es bien sabido, las revisiones sistemáticas bien elaboradas son formatos particularmente importantes para la audiencia médica profesional a la hora de hacer frente a la explosión de información en diferentes tópicos. Sin embargo, algunos autores han advertido que la calidad metodológica de la mayoría de las revisiones sistemáticas sobre los brotes de coronavirus fue y sigue siendo insatisfactoria, e incluso que las referidas a dicha entidad tienen mayor riesgo de mala calidad, posiblemente por la velocidad vertiginosa de las acciones necesarias para investigar, procesar los datos y difundir los resultados. Esto también llevó a tomar conciencia más cabal sobre la necesidad de una mejor alfabetización informacional y las estrategias que se requieren para una recepción y discusión más crítica de la información que se va publicando.

La terrible situación sanitaria global puso sobre la mesa otros varios dilemas y problemas éticos que ya eran conocidos. Sobre estos temas, recomendamos al lector el artículo publicado por Delia Sánchez en este mismo número de la RUC.

En paralelo, los gobiernos respondieron de diversas formas a la gran crisis científica ${ }^{(11)}$. Solo unos pocos, como Australia, destinaron fondos excepcionales para apoyar a la educación superior y las industrias intensivas con áreas de investigación aplicada. En EE. UU., en cambio, la mayoría de los proyectos de investigación se perdieron en un gran plan de rescate económico que costó 2.300 millones de dólares. Para agosto de 2020, un gran número de campus universitarios de EE. UU. y Europa comenzaban a reabrir sus puertas, a pesar de las crecientes tasas de infección. Otros países, que sufrían grandes brotes de contagios y tasas de muerte elevadas, como India y Brasil, por ejemplo, decidieron no reiniciar sus actividades de investigación en la misma medida. En Latinoamérica y el Caribe, y salvo muy contadas excepciones, el brutal impacto sanitario, social y económico se tradujo en recortes y/o ausencia de fondos incrementales dedicados a la investigación sobre COVID-19. Uruguay sufrió los impactos de la pandemia en forma tardía pero inexorable, con más de 6.000 muertes y 390.000 contagios a la fecha (https://www.gub.uy/sistema-nacional-emergencias/pagina-embebida/visualizador-casos-coronavirus-covid-19-uruguay). Como en otros lugares, los profesionales de la salud debieron tensar sus fuerzas, trabajando en condiciones de apremio, expuestos a un elevado riesgo de contagio y con la amenaza latente de saturación del sistema de cuidados críticos, mientras se coordinaba un plan de inmunización de alcance nacional.

A su vez, los efectores en salud y los organismos rectores nacionales debieron atender una demanda extraordinaria de nuevos procesos asistenciales, como el testeo masivo para SARSCoV-2, por ejemplo, mientras la academia analizaba la evidencia disponible y ensayaba nuevas investigaciones que daban soporte empírico a las respuestas sanitarias, en un escenario epidemiológico extremadamente cambiante. Este contexto abre más incertidumbre sobre los impactos específicos de la pandemia sobre las actividades locales de investigación.

Muchos pacientes con COVID-19 requirieron atención cardiológica, ya sea presencial o telefónica en domicilio, al borde de la cama, o requiriendo técnicas y procedimientos especializados en áreas de cuidados generales, intermedios o críticos. Trascurridos los primeros grandes brotes, la afectación miocárdica secundaria al COVID-19 resultó ser mucho más frecuente y grave de lo esperado, lo cual tiene implicancias pronósticas a mediano y largo plazo difíciles de predecir. Un metaanálisis reciente, dirigido a analizar las complicaciones cardiovasculares en 10.989 sujetos con COVID-19 incluidos en 35 estudios, demostró que se produjo lesión miocárdica aguda en uno de cada cuatro pacientes. Además, el ingreso a las UCI de estos pacientes fue 13,5 veces mayor y la mortalidad se multiplicó por 20 en comparación con los pacientes sin afectación cardíaca ${ }^{(15)}$. Las arritmias representaron el motivo de ingreso en casi $12 \%$ de los pacientes ingresados por COVID-19, con mayor exposición de los individuos 
mayores e hipertensos ${ }^{(16)}$. Además, la atención de los pacientes con patologías cardiológicas crónicas vio retrasados sus consultas clínicas, estudios y controles. Aquellos pacientes con formas potencialmente graves de la enfermedad coronaria se vieron en particular expuestos a impactos pronósticos imprevistos $^{(3,8)}$. Es probable que las políticas de contención y cierre relacionadas con la pandemia hayan influido directamente sobre la práctica clínica cardiológica. En Francia, por ejemplo, antes de las medidas restrictivas, las unidades de cuidados intensivos cardíacos admitían un promedio de 4,8 pacientes/ día por infarto agudo de miocardio o insuficiencia cardíaca, decayendo a 2,6 pacientes/día durante la aplicación de las restricciones ${ }^{(17)}$. Y muchas nuevas preguntas irán surgiendo a partir de las investigaciones relacionadas con los impactos cardiovasculares de la nueva infección global, en especial aquellas que magnifiquen el papel del daño lesional posCOVID sobre el sistema cardiovascular. Las respuestas a estas preguntas marcarán, al menos, la asistencia de los pacientes que sobrevivieron a una infección por coronavirus en los próximos años.

\section{Cambios en el proceso editorial y la modalidad de publicación}

La pandemia también trajo consigo algunos cambios positivos. Incluso cuando se cerraron las fronteras, surgieron y crecieron las colaboraciones internacionales, mayormente involucrando universidades médicas, gobiernos y empresas farmacéuticas $^{(11)}$. En cierta medida, el enorme monto de nueva información científica disponible, publicada en mayor parte en revistas de interés clínico general, y su impacto sobre la comunidad profesional y el público, han contribuido también a que la investigación y sus aplicaciones sean uno de los principales focos de las políticas públicas. Los investigadores comenzaron a compartir datos de manera más abierta, y muchos de ellos publicaron su trabajo en servidores de documentos preimpresos (preprints), un formato que solo utilizaban unas pocas disciplinas. La mayoría de las revistas hicieron que sus artículos referidos al COVID fueran de lectura gratuita. En muy poco tiempo, la cultura de la investigación se alejó de un paradigma que venía haciendo gran hincapié en la productividad ("publicar o morir", "to publish or to die") y pasó a debatir cuestiones más amplias y realistas, como el equilibrio entre el trabajo y la vida privada ("publicar mientras se vive", "to publish while living"). Con cierta perplejidad y demora, la comunidad científica pasó a ser más cautelosa y crítica con respecto a la relación entre los beneficios de la transferencia rápida de nuevos conocimientos y el daño potencial sobre la calidad de los artículos publicados, debido a los procesos de revisión acelerados. Habrá que esperar para saber si estos impactos pueden trascender en el tiempo.

Aunque ya hace casi 45 años que la Medicina se ha convertido en "un tema de titulares", los resultados de la investigación científica nunca habían tenido un impacto mediático tan rápido y explosivo como en esta pandemia. Las redes sociales se transformaron en voceros a tiempo real de los nuevos hallazgos. En contrapartida, la necesidad de expandir con celeridad el conocimiento sobre la infección por el nuevo coronavirus hizo que las exigencias de publicación se hicieran más laxas, y muchos artículos de escasa rigurosidad y dudosas garantías éticas fueron aceptados en pos de la velocidad de difusión; una ansiedad científica que, a riesgo de dañar la reputación de muchas revistas y medios de comunicación ante sus lectores, propició que la urgencia le ganara a la calidad; la nueva norma de las publicacio$n e s^{(14)}$. La atención de los medios se dirigió a los profesionales médicos, que informaban a diario sobre los avances científicos. Infectólogos, virólogos y epidemiólogos se convirtieron en nuevos influencers, y el público basó, al menos parte de sus opiniones, en información extraída de las redes. Sin embargo, las redes sociales y otros medios también pueden desinformar, por lo que la comunidad científica debe seguir actuando con cautela y firmeza, apuntalando la calidad de las fuentes de información y guiando la interpretación de los hechos científicos que se disponen y difunden a través de los espacios mediáticos.

Está claro que varias cuestiones al respecto de un nuevo panorama científico y editorial quedaron instaladas en la comunidad científica y la sociedad: ¿una vez que se salga de esta crisis, podrán sostenerse los cambios más positivos observados en la forma en que las revistas médicas afrontan las crisis de información? ¿Se podrá seguir publicando muchos artículos en modalidad de acceso gratuito que brinden información clave para la Salud Pública, ya sea sobre COVID-19 u otras enfermedades comunes y/o graves que ya conocemos? ¿Se puede y debe acelerar el proceso de revisión de los artículos de investigación originales, a riesgo de perder rigor y validez? ¿Pueden los consejos editoriales permitir que se dispongan más artículos en formato de preimpresos y menos originales debidamente revisados por pares? Las respuestas, que atañen de cerca a la SUC y a la $R U C$, seguramente estarán disponibles en un futuro próximo. 


\section{Bibliografía}

1. Sanchis J, Avanzas P, Bayes-Geniz A, Pérez de Isla L. In memoriam. Magda Heras i Fortuny, una huella imborrable en Revista Española de Cardiología. Rev Esp Cardiol. 2014; 67(10):867-8.

2. Reyes Caorsi W. Momento de decisiones en la Revista Uruguaya de Cardiología. Rev Urug Cardiol. 2016; 31(3):371-3.

3. Rodríguez-Leor O, Cid-Álvarez B, Pérez de Prado A, Rossello X, Ojeda S, Serrador A, et al; Grupo de Trabajo sobre Código Infarto de la Asociación de Cardiología Intervencionista de la Sociedad Española de Cardiología. Impacto de la COVID-19 en el tratamiento del infarto agudo de miocardio con elevación del segmento ST: la experiencia español. Rev Esp Cardiol. 2020; 73(12):994-1002. doi: 10.1016/j.recesp.2020.07.033

4. Lonis A, Moya J, Jalil JE, Ocaranza M, Rigotto A, Gabrielli L. Remodelado cardíaco en el modelo preclínico de falla cardíaca isquémica experimental por dislipidemia en el ratón SR-B1 KO/ ApoER6 1h/h. Rev Chil Cardiol. 2020; 39(1):24-33. doi:10.4067/s0718-85602020000100024

5. Pérez Zerpa D, Fernández A, Ríos F, Silva E, Lorente M, Arocena M, et al. Perfil clínico y etiológico de pacientes operados con endocarditis activa. Seguimiento a diez años. Rev Urug Cardiol. 2020; 5(2):169-79. doi: 10.29277/cardio.35.2.8

6. Scasso C, Medina J. Desafíos de las publicaciones científicas en la pandemia por el nuevo coronavirus (SARS-CoV-2). Rev Méd Urug 2020; 36(3):316-21.
7. Diéguez-Campa C, Pérez-Neri I, Reyes-Terán G, Flores-Apodaca I, Castillo-Ledón-Pretelini J, Mercado-Bautista O, et al. The 2020 research pandemic: A bibliometric analysis of publications on COVID-19 and their scientific impact during the first months. Arch Cardiol Mex. 2020. doi: 10.24875/ ACM.20000370

8. Monsuez J; Editors' Network of the National Societies of Cardiology Journals. COVID-19 and the heart: insights from the National Societies of Cardiology Journals. Eur Heart J. 2021; 42(39):4003-5. doi: 10.1093/eurheartj/ehab262

9. Böhm M, Ewen S, Schweitzer A, Katus H. Scientific publication activity during COVID-19 shutdown. Clin Res Cardiol. 2020; 109(12):1443-5. doi: 10.1007/s00392-020-01707-9

10. Forero-Peña D, Carrión-Nessi F, Camejo-Ávila N, Forero-Peña M. COVID-19 en Latinoamérica: una revisión sistemática de la literatura y análisis bibliométrico. Rev Salud Pública. 2020; 22(2):e216. doi: 10.15446/rsap.V22n2.86878

11. Lau J. Coronavirus crisis inspiring 'unprecedented' global research effort. Times Higher Education, 2020. Disponible en: https://www.timeshighereducation. com/news/coronavirus-crisis-inspiring-unprecedented-global-research-effort. [Consulta: 6 octubre 2021].

12. Staniscuaski F, Reichert F, Werneck F, de Oliveira L, Mello-Carpes P, Soletti R, et al; Parent in Science Movement. Impact of COVID-19 on academic mothers. Science. 2020; 368(6492):724. doi: 10.1126/science.abc2740 